\title{
Contaminación Acústica en el campus de la USMA
}

\section{Guillermo F. Burke Mena ${ }^{1, *}$ Fernando Lasso De La Vega ${ }^{2}$}

${ }^{1}$ Profesor, Facultad de Ingeniería y Tecnología, Universidad Católica Santa María la Antigua (USMA), Panamá, República de Panamá.

2 Estudiante, Facultad de Ingeniería y Tecnología, Universidad Católica Santa María la Antigua (USMA), Panamá, República de Panamá.

*Autor para correspondencia. Email: gburkem@usma.com.pa

Recibido: 19 de julio de 2017

Aceptado: 11 de octubre de 2017

\begin{abstract}
In the last decade at the World level, in the developed, underdeveloped and developing countries, the problem of Acoustic Contamination has intensified. The World Health Organization (WHO) revealed that at least 278 million people worldwide had moderate to profound hearing defects. Panama, at present, does not escape this reality and the competent authorities have revealed that at the moment that more than 300 thousand Panamanians are affected directly and indirectly by urban and industrial noises, only in the capital city. The WHO suggests that in order to develop the teaching-learning process correctly, the sound range in the school buildings should not exceed $55 \mathrm{dBA}$, and in public places, less than $70 \mathrm{dBA}$.
\end{abstract}

Keywords: Noise; Acoustics; Sound; Decibel; Hearing; Isosorality; Isolines.

\section{Resumen}

En la última década a nivel Mundial, en los países desarrollados, subdesarrollados y en vía de desarrollo, se ha intensificado la problemática de la Contaminación Acústica. La Organización Mundial de la Salud (OMS) reveló que en el mundo al menos 278 millones de personas presentaban defectos de audición de moderados a profundos. Panamá, actualmente, no escapa de esa realidad y las autoridades competentes han revelado que actualmente que más de 300 mil panameños son afectados directa e indirectamente por ruidos urbanos e industriales, solamente en la ciudad capital. La OMS sugiere que para que se desarrolle el proceso de enseñanza-aprendizaje correctamente, el rango de sonido en los predios del centro educativo no debe sobrepasar los $55 \mathrm{dBA}$, y en logares públicos, menor de $70 \mathrm{dBA}$.

Palabras Claves: Ruido; Acústica; Sonido; decibelio; Audición; Isosonoridad; Isolíneas. 
Invest. pens. crit. (ISSN 1812-3864)

Vol. 5, No. 3, septiembre-diciembre 2017

pp. 19-33

\section{Introducción}

La acústica es la ciencia que estudia las propiedades de las vibraciones de un medio susceptible de generar sonidos, infrasonidos o ultrasonidos, y de propagarlos. Es decir, estudia las ondas materiales denominadas sonidos audibles y no audibles, su producción, transmisión y percepción (Flores Castro \& Castillo, 2012).

El sonido está constituido por ondas tridimensionales propagándose en frentes esféricos (caso de no haber obstáculos) que se alejan del foco emisor. En general, los sonidos ambientales no están compuestos de frecuencias puras (ondas armónicas), sino que tiene una forma compleja la onda que se propaga, pero puede ser representada por la suma de varios términos o frecuencias armónicas (desarrollo de Fourier). El sonido, como onda longitudinal de presión, transporta energía que llega a nuestros oídos para provocar un desplazamiento del tímpano y el posterior movimiento de los huesillos situados en el oído medio (yunque, martillo y estribo), que transmiten la energía elástica a la linfa que la transforma en estímulo eléctrico neuronal para ser interpretado en el cerebro. Por ello, la sensación acústica no es función directa de la intensidad sonora (energía sonora por unidad de superficie perpendicular al frente de ondas), sino que depende de cada persona en particular. Existe un comportamiento medio de la población como respuesta a sonidos de diferentes frecuencias.

El ruido es el contaminante más común, y puede definirse como el conjunto de fenómenos vibratorios aéreos, percibidos e integrados por el sistema auditivo, que provocan en el ser humano, bajo ciertas condiciones, una reacción de rechazo en forma de molestia, fatiga o lesión (Villarreal, Castillo, Muñoz, Toral, \& Flores Castro, 2013).

En general, el campo de audición del hombre está comprendido entre $20 \mathrm{~Hz}$ y $20000 \mathrm{~Hz}$. Cada frecuencia tiene su propio umbral de audición. El umbral del dolor o nivel sonoro cuya intensidad produce dolor esta alrededor de los $120 \mathrm{dBA}$.

El ruido según la OMS puede causar una minusvalía auditiva, dentro de las normas ISO (International Standar Organization), que es el deterioro auditivo severo que puede afectar la las actividades cotidianas del individuo. La exposición prolongada a ruidos de intensidad elevada origina una fatiga auditiva, pero si los ruidos exceden los $80 \mathrm{dBA}$. El deterioro auditivo puede ocurrir en distintos lugares (puestos de trabajo, centros de estudio, comunidad, etc.), el cual el ruido descontrolado esté frecuentemente presente, y puede causar efectos negativos sobre los individuos, como: perturbación del sueño, fatiga, depresión, bajo rendimiento, efectos cardiovasculares, etc.

\section{Nociones Acústica}

El oído humano: Órgano corporal o vestibulococlear del ser humano, cuya función principal es escuchar los sonidos y mantener el equilibrio de los mismos. Éste órgano está dividido en tres partes: oído externo, que percibe, capta y reorienta las vibraciones sonoras hacia el oído medio; oído medio, transforma las ondas sonoras en vibraciones mecánicas; y oído interno las vibraciones mecánicas son transformadas en señales nerviosas. 
Invest. pens. crit. (ISSN 1812-3864)

Vol. 5, No. 3, septiembre-diciembre 2017

pp. $19-33$

La sensación auditiva que se percibe en el oído se le denomina sonido. Cuando una diferencia de presión hace que las moléculas de aire que nos rodea vibren y llegan al sistema auditivo, propagadas en un medio (gas, líquido o sólido), podremos afirmar que es un sonido. Para que exista el sonido de haber una fuente de emisión, un medio de propagación y un receptor (Kogan Musso, 2004).

La acústica es la ciencia que estudia las propiedades de las vibraciones de las partículas de un medio susceptible de engendrar sonidos, infrasonidos o ultrasonidos; de propagarlos y de hacerlos perceptibles. Tomando en cuenta los efectos sobre el ser humano, algunos sonidos son catalogados como ruido. El ruido se puede definir como el conjunto de sonidos percibidos por el sistema auditivo humano que provocan, en el receptor, molestia, lesión o fatiga (Flores Castro \& Castillo, 2012).

El ser humano tiene un campo audible entre $20 \mathrm{~Hz}$ y $20 \mathrm{kHz}$, los sonidos en este campo audible que estén por debajo de este rango, se le denominan infrasonidos; y los que sobrepasan el rango audible, se le denominan ultrasonidos (ambos no son audibles para el oído humano).

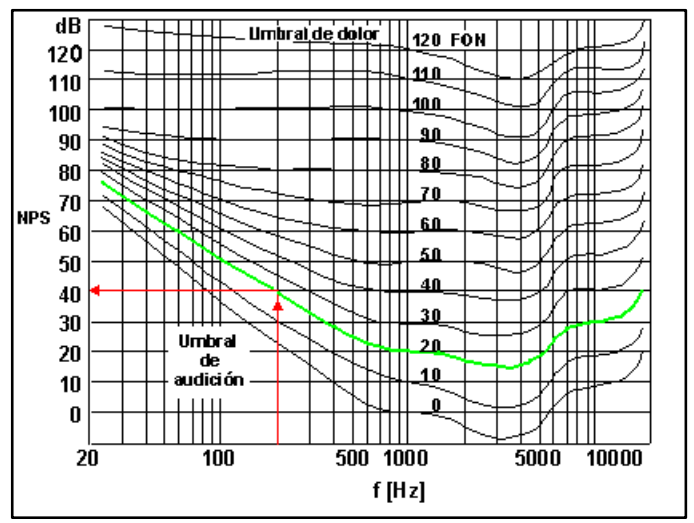

Ilustración 1. Umbrales Auditivos del Oído Humano (Miyara, 2003).

El umbral de audición, o el nivel de presión sonora (NPS) que nuestro oído puede percibir, dependen de la frecuencia con que se emite el sonido; es decir que la respuesta del oído no es la misma en todas las frecuencias (ilustración 1).

La exposición prolongada a sonidos de intensidad elevada (ruido) origina fatiga auditiva que es recuperable, pero si los ruidos exceden los $80 \mathrm{~dB}$ en ponderación " $\mathrm{A}$ " (asimila el oído humano) y se prolonga por meses la exposición, se origina una sordera denominada neurosensorial, totalmente irreversible. 
Invest. pens. crit. (ISSN 1812-3864)

Vol. 5, No. 3, septiembre-diciembre 2017

pp. $19-33$

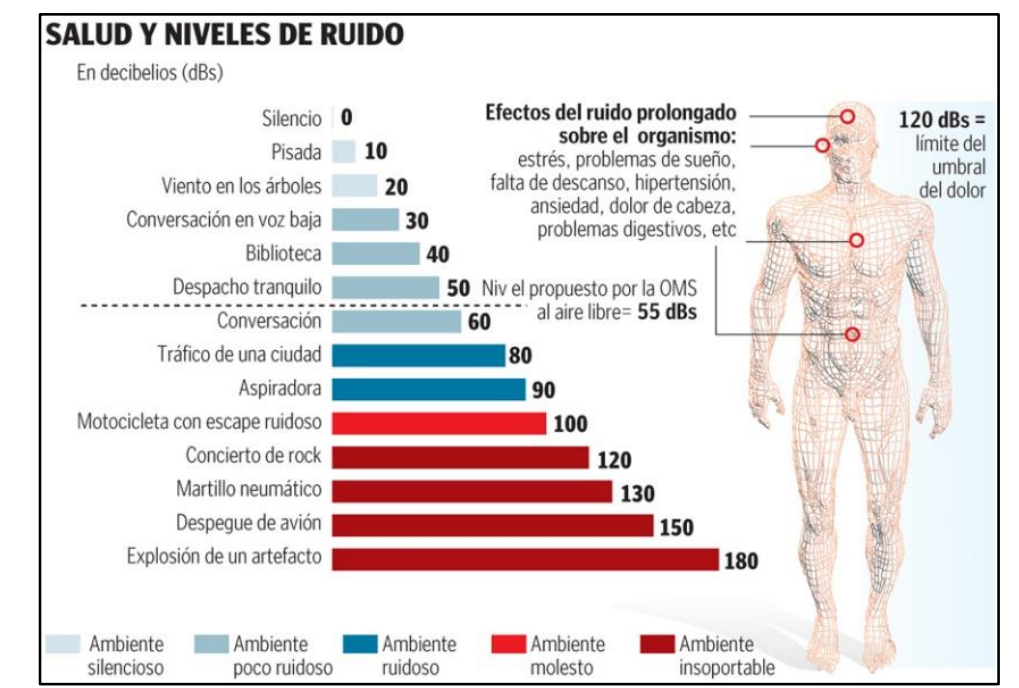

Ilustración 2. Salud y Niveles de Ruido (Armas Varquez, 2013).

La molestia por el ruido puede definirse como un sentimiento desagradable, que produce efectos perjudiciales en la salud de una persona. Es fácil saber cuándo una persona está molesta por el ruido, pero si es difícil saber de cuantificar el grado de molestia (Flores Castro \& Castillo, 2012).

\section{Medición Del Ruido}

Los ruidos se pueden clasificar de varias maneras: por su carácter personal (ruido constante, intermitente, fluctuante, de impacto, periódico, etc.); por sus características espectrales (tono puro, ruido de banda ancha, estrecha, banda blanca, etc.); por su naturaleza (fuente); por su nivel sonoro (alto, medio, bajo); etcétera (Kogan Musso, 2004).

Para medir el ruido, existen muchas formas, pero todas requieren de un indicador, el cual pueden permitir cuantificar el sonido que uno desea captar o medir. De todos los indicadores, el que más se utiliza es el Nivel de Presión Sonora ( $\left.L_{P}\right)$, que determina la intensidad del sonido que percibe una persona debido a su presión sonora instantánea. Su unidad de medida en el Bel (B), en honor al inventor escocés Alexander Graham Bell, pero por escala e utiliza el prefijo “deci” y su expresión matemática es:

$$
L_{P}=20 \log \left(\frac{p}{p_{r}}\right)(d B)
$$

Ecuación 1

Donde $p$ es la presión sonora en un instante y $p_{r}$ es la presión de referencia y tiene un valor constante de $20 \mu \mathrm{Pa}$, y es la mínima presión que puede percibir el oído humano.

Cuando el ruido es constante o de larga duración, periódico, o similar, se puede utilizar el Nivel Sonoro continuo Equivalente $\left(\mathrm{L}_{\mathrm{eq}}\right)$, que sería el nivel de ruido medido en un lapso de tiempo y su expresión matemática es:

$$
L_{e q}=10 \log \left(\frac{1}{T} \cdot \int_{0}^{T} \frac{p^{2}(t)}{p_{r}^{2}} d t\right)(d B)
$$

Ecuación 2 
Invest. pens. crit. (ISSN 1812-3864)

Vol. 5, No. 3, septiembre-diciembre 2017

pp. 19-33

Donde $p(t)$ es la presión sonora en función del tiempo y Tes el período de la medición.

El sonómetro es un instrumento que se utiliza para medir el nivel de presión sonora. En general, los sonómetros están constituidos por un micrófono, un preamplificador, un amplificador, circuito de ponderación de frecuencia, control de nivel, integrados de tiempo y dispositivo de lectura. Los sonómetros tienen la facilidad de ponderarse, según el rango de frecuencias de la onda sonora que incide en el micrófono. Los cuatro tipos de ponderación son:

- Ponderación A: esencial para medir ruidos de intensidad baja, es la más utilizada, ya que es la que más se asemeja a la respuesta del oído humano.

- Ponderación B: Asemeja el oído humano, pero para frecuencias de nivel medio (no es muy empleada)

- Ponderación C: Creado para modelar la respuesta del oído ante sonidos de gran intensidad.

- Ponderación D: Utilizada para medir niveles de intensidad sonora provocada por aviones o similares.

- Ponderación U: Ponderación especial, es utilizada para medir sonidos audibles en presencia de ultrasonidos.

\section{Amparos Legales sobre el Ruido en Panamá}

Existen varios amparos legales vigentes, sobre ruido, en el cual se puede recurrir para poner quejas, en caso de que se encuentre molestia del mismo:

- $\quad$ LA CONSTITUCIÓN DE LA REPÚBLICA DE 1972.

- Artículo 105: Es función esencial del Estado velar por la salud pública, que incluye la responsabilidad de asegurar el derecho que tiene el individuo a la promoción, prevención y rehabilitación de la salud.

- Artículo 115: El Estado y todos los habitantes del territorio nacional tienen el deber de proporcionar un desarrollo social y económico que prevenga la contaminación del ambiente, mantenga el equilibrio ecológico y evite la destrucción de los ecosistemas.

- Ley 66 de 10 de noviembre de 1947: Por la cual se aprueba el Código Sanitario.

- Artículo 88: Son actividades sanitarias locales en relación con el control del ambiente: 1. Dictar las medidas tendientes a evitar o suprimir las molestias públicas, como ruidos, olores desagradables, humos, gases tóxicos, etc.; 2. Ubicar en zonas determinadas las industrias peligrosas o molestas, los establos y pesebreras, etc.

- Ley No 41 de 1 de julio de 1998: Ley General de Ambiente de la República de Panamá.

- Artículo 4: Son principios y lineamientos de la política nacional del ambiente, los siguientes: 1. Dotar a la población, como deber del Estado, de un ambiente saludable y adecuado para la vida y el desarrollo sostenible.

- Decreto Ejecutivo No 306 de 4 de septiembre de 2002: Reglamento para el Control de los Ruidos en Espacios Públicos, Áreas Residenciales o de Habitación, así como en Ambientes Laborales. Modificado por el Decreto Ejecutivo No 1 de 15 de enero de 2004.

- Horario de 6:00 a.m. a 9:59 p.m.: Nivel sonoro máximo de 60 dBA. 
Invest. pens. crit. (ISSN 1812-3864)

Vol. 5, No. 3, septiembre-diciembre 2017

pp. $19-33$

- Horario de 10:00 p.m. a 5:59 a.m.: Nivel sonoro máximo de 50 dBA.

- Reglamento Técnico DGNTI-COPANIT 44-2000: Advierte que la exposición permisible para jornadas de 8 horas de trabajo, estará en función del ruido.

$-8 \mathrm{~h}(85 \mathrm{dBA})$

- $7 \mathrm{~h}(86 \mathrm{dBA})$

- $45 \min (102 \mathrm{dBA})$
$-6 \mathrm{~h}(87 \mathrm{dBA})$

$-5 \mathrm{~h}(88 \mathrm{dBA})$

- $30 \min (105 \mathrm{dBA})$
- $4 \mathrm{~h}(90 \mathrm{dBA})$

- 3 h (92 dBA)

- $12 \min (110 \mathrm{dBA})$
- $2 \mathrm{~h}(95 \mathrm{dBA})$

- $1 \mathrm{~h}(100 \mathrm{dBA})$

$-7 \min (115 \mathrm{dBA})$

\section{- DECRETOS DEL MUNICIPIO DE PANAMÁ}

- Acuerdo No 57 del 23 de mayo de 2006, por el cual se reglamenta las actividades de la industria de la construcción que generen ruidos perjudiciales para la salud.

- Decreto No 4113 de 26 de junio de 2006, por la cual se regulan las emisión de ruido en la Ciudad de Panamá.

Actualmente en el campus central de la USMA no se han realizado estudios de impacto ambiental de ruido, para saber si la institución cuenta con el ambiente propicio que la OMS y el Decreto Ejecutivo 306 de 2002, de la República de Panamá, sugieren para los centros de estudios. Este trabajo tiene la finalidad de medir el nivel de sonido que actualmente se frecuentan en las aulas de clases y los predios de la USMA, para saber si se cumple o no las condiciones de estudios como centro educativo, identificar las posibles barreras de ruido y posibles fuentes de origen.

\section{Métodos y Materiales}

\section{Zona de Estudio}

La Universidad Católica Santa María La Antigua de Panamá, centro particular de estudios superiores, está ubicada al costado de una de las vías principales de la ciudad de Panamá (sector urbano), específicamente en las coordenadas $9^{\circ} 1^{\prime}$ '46” de Latitud Norte y $79^{\circ} 31$ ' 15” de Longitud Oeste. Su ubicación pertenece al corregimiento de Bethania, del distrito de Panamá, Provincia de Panamá; donde el índice de precipitación estandarizado (SPI) es Ligeramente Seco, además tiene un clima tropical de tipo sub-ecuatorial caracterizado por una prolongada estación lluviosa de mayo a diciembre y una corta estación seca de enero a abril. La precipitación anual promedio está cerca de los $2000 \mathrm{~mm}$, una humedad relativa promedio de $75 \%$ y una temperatura promedio de $27^{\circ} \mathrm{C}$, con máximas absolutas de hasta $39^{\circ} \mathrm{C}$ y mínimas de $20{ }^{\circ} \mathrm{C}$.

\section{Operaciones de Campo}

- Contexto: Esta vertiente cualitativa se realizó con un plan de estudio específico de las áreas de posibles exposiciones ruido en la USMA, como áreas sociales, zonas aledañas y aulas de clases, comparándolas con un punto patrón. Cada punto patrón se georreferenció con la ayuda de un GPS de la Marca Garmin modelo etrex 10 (ilustración 3). 
Invest. pens. crit. (ISSN 1812-3864)

Vol. 5, No. 3, septiembre-diciembre 2017

pp. 19-33

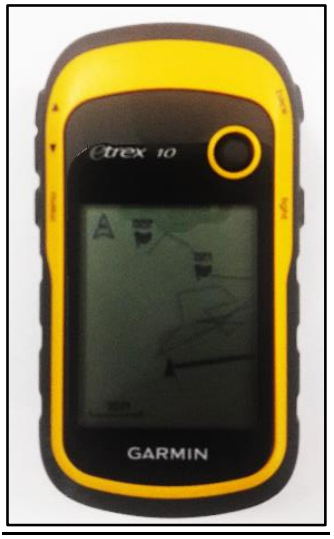

\section{Ilustración 3. Sistema de Posición Global GPS}

Con los resultados adquiridos se realizaron mapas de superficie de isosonoridad superpuestos en un mapa satelital de la USMA, con la finalidad de estudiar las posibles fuentes de sonidos extremos y las direcciones de barreras de sonido.

- Variable: La única variable a medir es la intensidad de presión sonora equivalente contra tiempo de muestreo.

- Equipo de Recolección: Existen diversas formas de medir los niveles de sonoridad, pero la más recomendada, por las entidades internacionales y nacionales, es el nivel de presión sonora al que está expuesto un individuo que es el nivel de presión sonora equivalente, utilizando la ponderación A (Flores Castro \& Castillo, 2012). Para ello se contará con 10 sonómetros PCE-322 A (ilustración 7) con las siguientes características:

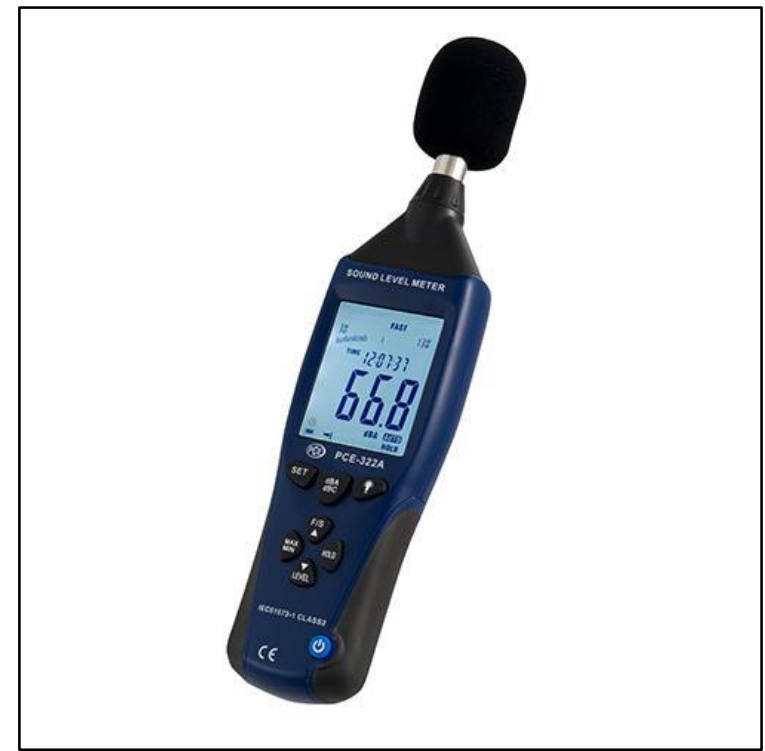

Ilustración 4. Sonómetros PCE-322 A

- Rango automático 30...130 dB

- Rangos manuales $30 \mathrm{~dB}$ a $80 \mathrm{~dB} / 50 \mathrm{~dB}$ a $100 \mathrm{~dB} / 80 \mathrm{~dB}$ a $130 \mathrm{~dB}$ 
Invest. pens. crit. (ISSN 1812-3864)

Vol. 5, No. 3, septiembre-diciembre 2017

pp. $19-33$

- Resolución $0,1 \mathrm{~dB}$

- Precisión $\pm 1,4 \mathrm{~dB}$

- Mostrar actualización Cada 0,5 s

- Frecuencia $31,5 \mathrm{~Hz}$ a $8 \mathrm{kHz}$

- Logger de datos 262100 puntos

- Valoración A, C

- Pantalla Gran pantalla LCD con gráfico de barras

- Temperatura operativa $0{ }^{\circ} \mathrm{C}$ a $40{ }^{\circ} \mathrm{C},<80 \%$ H.r.

- Alimentación Pila de $9 \mathrm{~V}$ (duración 30h uso continuo) o adaptador de red para mediciones prolongadas

- El cual sirven para medir el nivel de presión sonora en función del rango de frecuencia de la onda sonora incide sobre el micrófono.

- Procedimiento: Estos sonómetros fueron ubicados estratégicamente, funcionando en tiempo corrido durante horas de la mañana, tarde y noche (horario de clases regular), y estrictamente georeferenciados mediante un sistema de posición global (GPS), para facilitar el desarrollo de los mapas de isosonoridad.

- Análisis: La adquisición diaria de las intensidades de sonido de los 10 sonómetros se diferenció en datos matutinos, vespertinos y nocturnos (cuatrimestral y anual), realizando un análisis de varianza ANOVA para una mejor comparación (Castro, 2005). Gracias a su posición georeferenciada fue fácilmente mapeado con isolíneas de intensidad sonora, con ello se procedió con el análisis sistemático cuantitativo para establecer las fuentes de ruido y la dirección de propagación de las barreras de sonidos. Internamente para análisis cuantitativo interno, se realizarán gráficas mensuales para un mejor manejo de la posible problemática.

\section{Resultados, Discusión}

Para establecer una red o puntos de muestreo se realizó un estudio de georreferenciación en el área de estudio, para poder determinar los puntos exactos de captación de intensidad de nivel sonoro equivalente de dichos lugares. A cada punto de medición se le midió sus coordenadas con un GPS, Garmin etrex 10, y se le identificó con un código "S" y el número al código se le iba asignando a medida que se iban colocando los sonómetros en dichos lugares específicos (tabla 1).

Tabla 1. Georreferenciación de los Sonómetros

\begin{tabular}{|c|c|c|c|c|c|c|c|}
\hline $\begin{array}{c}\text { Códig } \\
\text { o }\end{array}$ & $\begin{array}{c}\text { Nombre } \\
\text { Específico }\end{array}$ & $\begin{array}{c}\text { Posición } \\
\text { X }\end{array}$ & $\begin{array}{c}\text { Posición } \\
\text { Y }\end{array}$ & $\begin{array}{l}\text { Códi } \\
\text { go }\end{array}$ & $\begin{array}{c}\text { Nombre } \\
\text { Específico }\end{array}$ & $\begin{array}{c}\text { Posición } \\
\text { X }\end{array}$ & $\mathrm{Y}$ \\
\hline S01 & S01 (R & $\begin{array}{c}662634,15 \\
94\end{array}$ & $\begin{array}{c}998365,9 \\
482\end{array}$ & S06 & $\begin{array}{c}\text { S06 } \\
\text { (biblioteca) }\end{array}$ & $\begin{array}{c}662582,3 \\
888\end{array}$ & $\begin{array}{c}998480,0 \\
207\end{array}$ \\
\hline S02 & $\begin{array}{r}\mathrm{S} 02(\mathrm{~S} \\
\mathrm{Pr}\end{array}$ & $\begin{array}{c}662536,5 \\
711\end{array}$ & \begin{tabular}{|c|}
998481,67 \\
83
\end{tabular} & S07 & $\begin{array}{r}\text { S07 } \\
\text { SOC }\end{array}$ & $\begin{array}{c}662502,19 \\
59 \\
\end{array}$ & $\begin{array}{c}998372,7 \\
866\end{array}$ \\
\hline S03 & S03 (C & $\begin{array}{l}662546,0 \\
095\end{array}$ & $\begin{array}{c}998413,51 \\
58\end{array}$ & S08 & S08 (Capilla) & $\begin{array}{c}662619,15 \\
65\end{array}$ & $\begin{array}{c}998450,6 \\
775\end{array}$ \\
\hline S04 & $\begin{array}{c}\text { S04 } \\
\text { (Mantenimiento) }\end{array}$ & $\begin{array}{c}662514,72 \\
41\end{array}$ & $\begin{array}{c}998446,5 \\
678\end{array}$ & S09 & S09 (VIP) & $\begin{array}{c}662756,2 \\
432\end{array}$ & $\begin{array}{c}998436,0 \\
236\end{array}$ \\
\hline
\end{tabular}


Invest. pens. crit. (ISSN 1812-3864)

Vol. 5, No. 3, septiembre-diciembre 2017

pp. 19-33

\begin{tabular}{|l|c|c|c|c|c|c|c|}
\hline \multirow{2}{*}{ S05 } & \multirow{2}{*}{ S05 (gimnasio) } & $\mathbf{6 6 2 6 4 8 , 1 0}$ & $\mathbf{9 9 8 5 4 2 , 9}$ & \multirow{2}{*}{ S10 } & S10 (Edificio & $\mathbf{6 6 2 4 8 7 , \mathbf { 1 1 }}$ & $\mathbf{9 9 8 4 7 5 , 9}$ \\
& & $\mathbf{5 8}$ & & $\mathrm{G})$ & $\mathbf{8 6}$ & $\mathbf{4 8 2}$ \\
\hline
\end{tabular}

Estos sonómetros fueron ubicados en un mapa georreferenciado a un sistema interactivo Google Maps, (ilustración 8 y 9) con el fin de establecer la ubicación espacial de los mismos y poder establecer un estudio específico con mayor eficiencia.

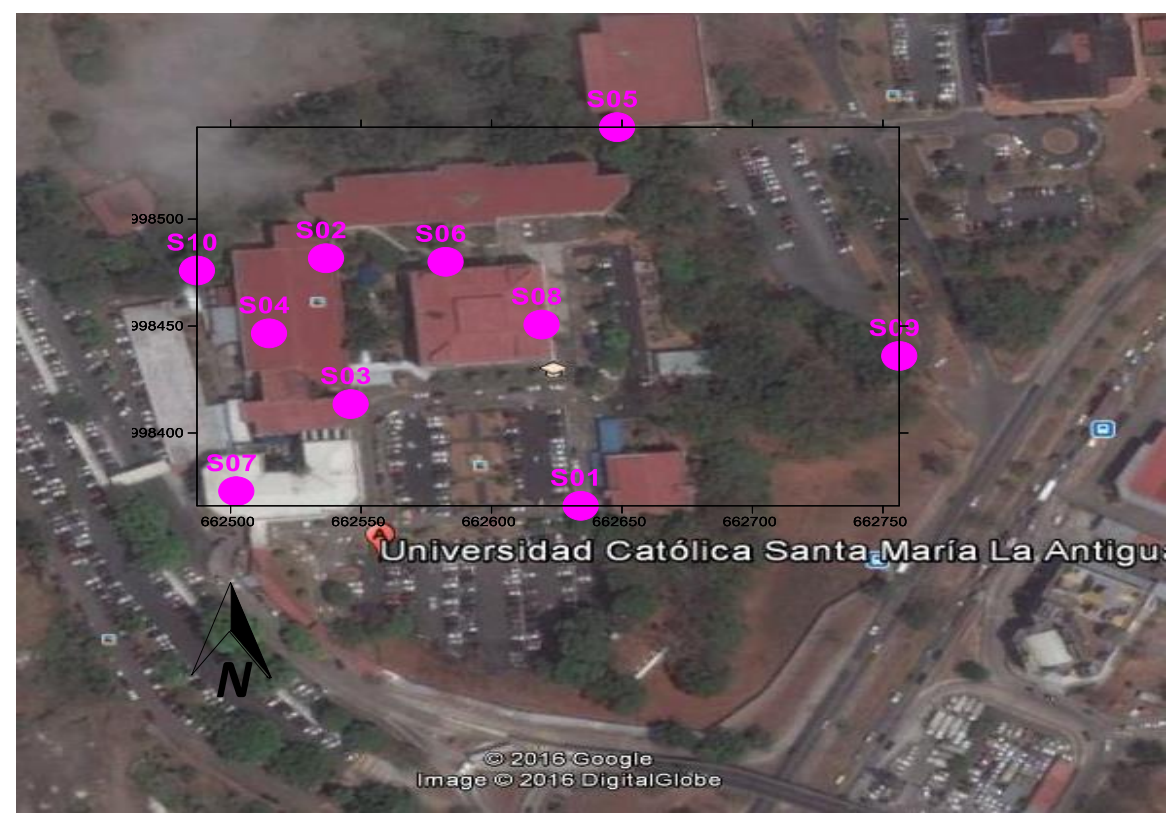

Ilustración 5. Ubicación Espacial de la distribución de los Sonómetros. (Fuente: Mapa Interactivo Google Maps)

La adquisición diaria de las intensidades de nivel sonoro equivalente de los 10 sonómetros, se hicieron de manera automática en intervalos de 30 segundos, ya que los sonómetros tienen la capacidad de captar 262100 datos programados (memoria interna); la extracción de los datos de los sonómetros se realizaron cada 15 días (ilustración 6), con el fin de monitorear los equipo de medición y salvaguardar los datos de la investigación. 

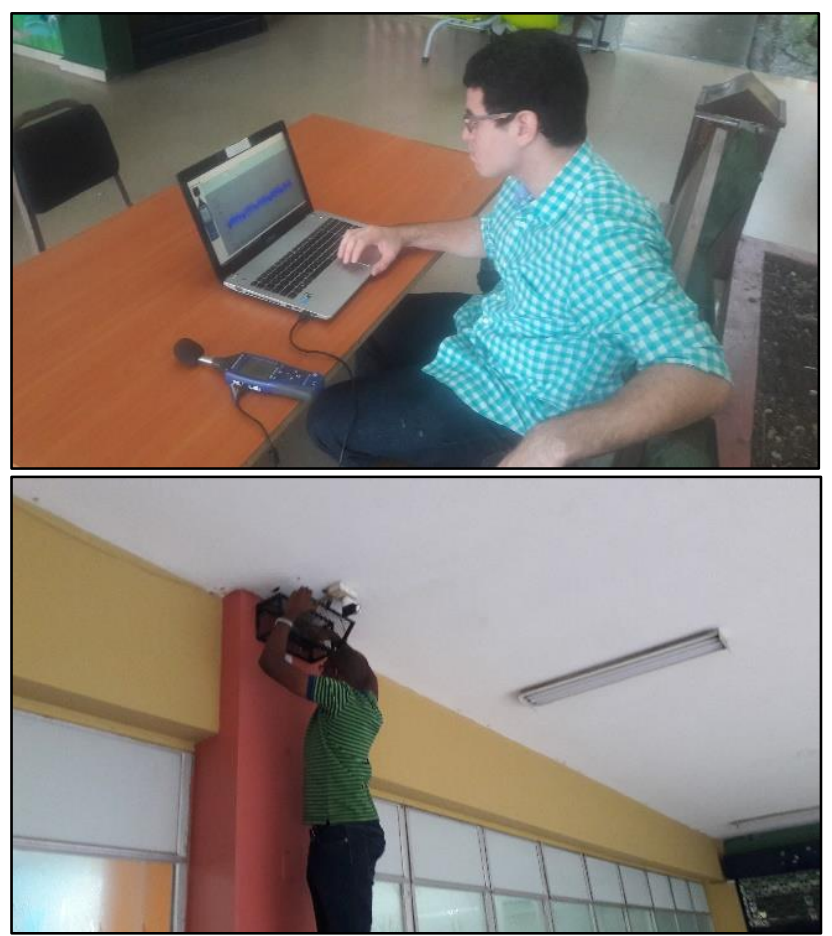

Ilustración 6. Extracción de Datos de los Sonómetros

Se diferenció en datos matutinos, vespertinos y nocturnos (anual), realizando un análisis estadístico específico, para una mejor comparación. Gracias a su posición georreferenciada fue fácilmente mapeado con isolíneas de intensidad sonora, con ello se procedió con el análisis sistemático cuantitativo para establecer las fuentes de ruido y la dirección de propagación de las barreras de sonidos.

Los resultados de los niveles sonoros equivalentes (promedio) por turno durante el año, se presentan en la siguiente tabla de datos:

Tabla 2. Nivel Sonoro Equivalente Anual por turno.

\begin{tabular}{|c|c|c|c|c|c|c|c|c|c|c|}
\hline & $\begin{array}{c}\text { 2do } \\
\text { Cuatr } \\
\text { AM('16 } \\
\text { ) }\end{array}$ & $\begin{array}{c}\text { 2do } \\
\text { Cuatr } \\
\operatorname{PM(}\left({ }^{\circ} 16\right. \\
)\end{array}$ & $\begin{array}{c}\text { 2do } \\
\text { Cuatr } \\
\text { Noche(“ } \\
16)\end{array}$ & $\begin{array}{c}3 \text { er } \\
\text { Cuatr } \\
\operatorname{AM(}\left({ }^{\circ} 16\right. \\
)\end{array}$ & $\begin{array}{c}\text { 3er } \\
\text { Cuatr } \\
\operatorname{PM('16} \\
\text { ) }\end{array}$ & $\begin{array}{c}\text { 3er } \\
\text { Cuatr } \\
\text { Noche( } \\
16)\end{array}$ & $\begin{array}{c}\text { 1er } \\
\text { Cuatr } \\
\operatorname{AM}\left({ }^{\circ} 17\right. \\
)\end{array}$ & 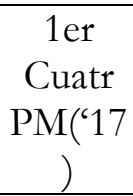 & $\begin{array}{c}\text { 1er } \\
\text { Cuatr } \\
\text { Noche(‘ } \\
17)\end{array}$ & \\
\hline S01 (Rectoría) & 65,14 & 65,12 & 61,89 & 65,28 & 65,32 & 62,49 & 64,74 & 64,48 & 61,26 & E \\
\hline $\begin{array}{l}\text { S02 (Salón de } \\
\text { Prof) }\end{array}$ & 56,17 & 56,10 & 53,63 & 57,26 & 57,88 & 56,04 & 57,48 & 57,09 & 55,19 & 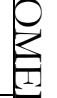 \\
\hline S03 (Cajero) & 60,06 & 59,76 & 58,05 & 60,03 & 60,19 & 58,41 & 58,81 & 57,72 & 56,52 & $\underline{\underline{n}}$ \\
\hline $\begin{array}{c}\mathrm{S} 04 \\
\text { (Mantenimiento) }\end{array}$ & 67,45 & 70,79 & 65,72 & 67,64 & 68,97 & 64,36 & 65,23 & 70,91 & 66,82 & 2 \\
\hline S05 (gimnasio) & 57,90 & 59,50 & 61,58 & 58,77 & 59,28 & 60,48 & 56,51 & 57,05 & 58,92 & E \\
\hline
\end{tabular}


Invest. pens. crit. (ISSN 1812-3864)

Vol. 5, No. 3, septiembre-diciembre 2017

pp. 19-33

\begin{tabular}{|c|c|c|c|c|c|c|c|c|c|}
\hline S06 (biblioteca) & 61,22 & 60,23 & 57,04 & 62,23 & 63,63 & 61,83 & 64,01 & 62,37 & 57,85 \\
\hline S07 (área social) & 64,24 & 65,10 & 58,07 & 66,68 & 67,73 & 62,84 & 64,86 & 65,12 & 59,09 \\
\hline S08 (Capilla) & 62,62 & 62,87 & 61,92 & 61,73 & 62,83 & 60,52 & 63,67 & 64,61 & 63,20 \\
\hline S09 (VIP) & 67,56 & 68,03 & 65,21 & 65,97 & 65,88 & 63,80 & 64,31 & 63,78 & 62,46 \\
\hline S10 (Edificio G) & 63,39 & 65,44 & 62,36 & 61,38 & 63,42 & 60,58 & 61,66 & 63,69 & 60,40 \\
\hline
\end{tabular}

Con los datos adquiridos, se procedió a realizar un gráfico de superficie de isosonoridad equivalente, en la ilustración 7 se presenta este tipo de gráfico para el turno matutino anual, así poder realizar un análisis específico de la fenomenología acústica en el área.

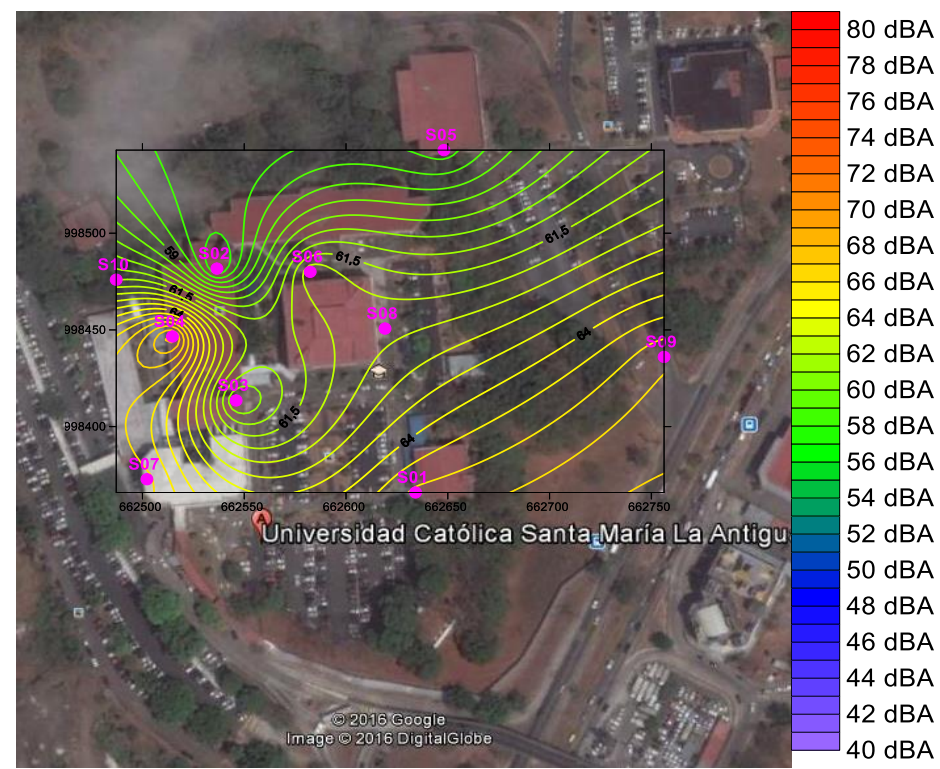

Ilustración 7. Gráfica de Promedio Nivel Sonoro Equivalente Anual 2016 - 2017 del turno matutino.

En este gráfico se puede determinar que en promedio matutino del nivel sonoro equivalente en el Campus central de la USMA es de $(62,66 \pm 0,01) \mathrm{dBA}$; marcando un nivel aceptable para área pública, según las normas panameñas, pero las recomendadas por la OMS para áreas educativas, sobrepasa los niveles permisibles. Mientras que su flujo de sonido muestra una barrera de sonido proveniente de la Avenida Ricardo J. Alfaro, con una dirección del sureste hacia noroeste. También se puede observar que dentro del campus universitario hay cuatro puntos de origen de sonido, S06, S02, S04 y S03. Estos lugares son, respectivamente, Biblioteca, Salón de Prof., Mantenimiento y Cajero; donde se produce mayor intensidad de sonido que el proveniente de la Avenida Ricardo J. Alfaro, además que hay una tendencia, en el área social del sonómetro S07 “área social”, en un aporte de nivel sonoro considerable.

Siguiendo con las gráficas de isosonoridad, en la ilustración 8 se muestra el promedio vespertino del nivel sonoro equivalente de $(63,17 \pm 0,01) \mathrm{dBA}$, con un comportamiento similar al matutino (ilustración 7). 


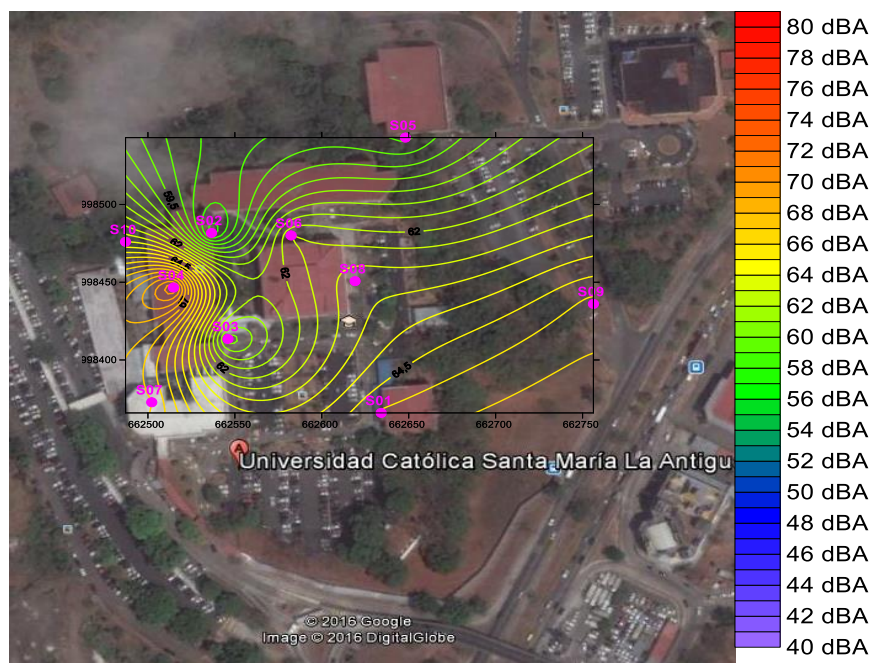

Ilustración 8. Gráfica de Promedio Nivel Sonoro Equivalente Anual 2016 - 2017 del turno Vespertino.

Mientras que en el turno nocturno, la ilustración 9 muestra que el promedio del nivel sonoro equivalente es de $(60,57 \pm 0,01) \mathrm{dBA}$, en el gráfico se aprecia un flujo sonoro, donde la propagación de sonido de fondo, se nota proveniente del este hacia el oeste del campus, pero en la zona central del campus se ve mucha generación de sonido intenso, por actividades realizadas en el campus finalizando el 2016.

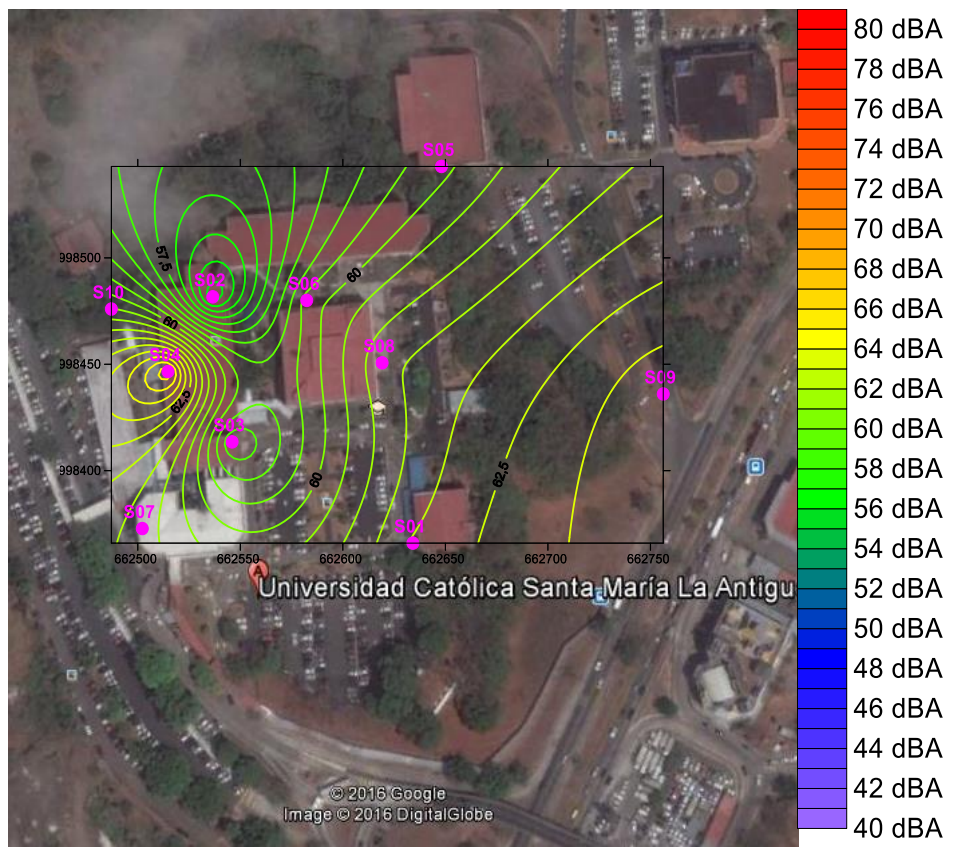

Ilustración 9. Gráfica de Promedio Nivel Sonoro Equivalente Anual 2016 - 2017 del turno Vespertino. 
Invest. pens. crit. (ISSN 1812-3864)

Vol. 5, No. 3, septiembre-diciembre 2017

pp. 19-33

Haciendo un análisis cuantitativo general del nivel sonoro equivalente en todo el año, podemos decir que en la mayoría del tiempo, incluyendo los horarios académicos, el campus central de la USMA tiene ruido tolerable, dentro de las normas que rigen en la República de Panamá, y si sobrepasa los 70 dBA, sólo el 12\% de las veces y de manera intermitente (ilustración 10), arrojando un promedio anual de $(62,35 \pm 0,01) \mathrm{dBA}$. Si el rango entre ambiente silencioso y ruidoso está entre $55 \mathrm{dBA}$ y $70 \mathrm{dBA}$, significa que las USMA está en el rango medio, entre silencioso y ruidoso, basado en las recomendaciones de la OMS.

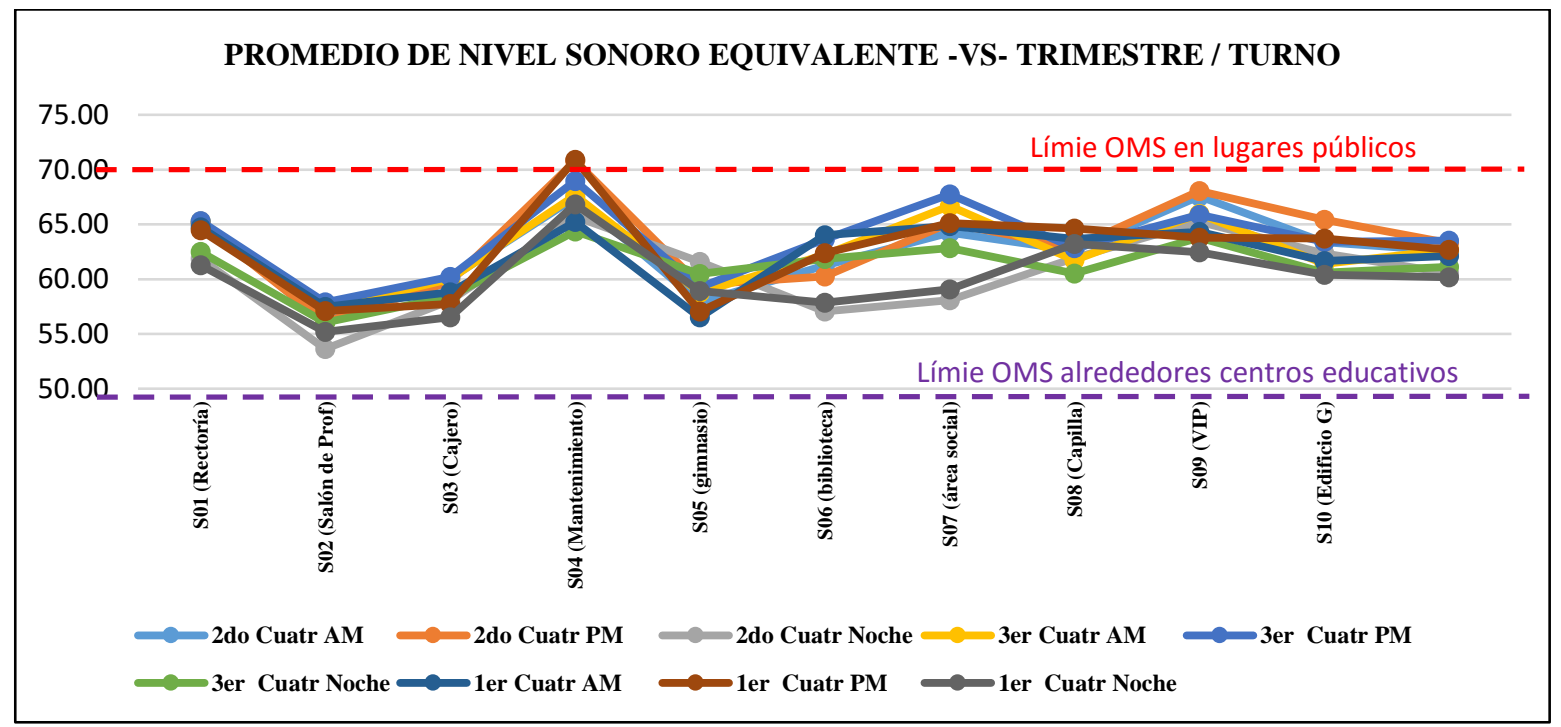

Ilustración 10. Nivel Sonoro Equivalente Anual en el Campus Central de la USMA

\section{Recomendaciones}

El ruido es una de las consecuencias inherentes de la productividad de una empresa. Para prevenir la posible producción de ruido en el Campus de la USMA, se recomienda:

- Comunicar a los Autoridades competentes de impacto ambiental de la USMA, sobre la sensitiva problemática de contaminación acústica en el campus, mostrándole los resultados de su propagación y fuentes primarias de ruido, para establecer un plan de posible mitigación del mismo.

- Efectuar un programa de sensibilización contra el ruido, en especial para la comunidad educativa, cómo prevenirlos y sus efectos en el deterioro de la salud y la productividad; ya que por análisis estadístico, se determinó que su modo de hablar no es tan moderado, y es causal ruido primario dentro del Campus.

- Proponer a las autoridades de la USMA que coloquen mensajes de prevención, control, saneamiento y reducción eficaz del ruido mediante anuncios, letreros banners, comunicación impresa y/o digital, congresos, charlas, entre otras. Así se reducirá posibles afectaciones auditivas de la comunidad educativa usmeña. 
Invest. pens. crit. (ISSN 1812-3864)

Vol. 5, No. 3, septiembre-diciembre 2017

pp. $19-33$

- Monitoreo de las maquinarias que sean potenciales fuente de ruido inherente, que podrían llegar a ser molestosos.

- Darle seguimiento al estudio para mejor monitoreo de las fuentes de ruido detectadas. También un estudio de saneamiento acústico en la Biblioteca de la USMA, ya que por comentarios de la comunidad usmeña, señalan que dentro de la Biblioteca de la USMA no se cumple con las normas ni con el comportamiento que se debe tener dentro de la Biblioteca. La OMS recomienda que dentro de la Biblioteca debe haber un Nivel sonoro equivalente no mayor a $40 \mathrm{dBA}$.

\section{Bibliografía}

Armas Vazquez, A. (8 de Noviembre de 2013). instituto salud y saber.inc. Obtenido de https://institutosaludysaber.files.wordpress.com/2013/11/ruido.jpg

Ballesteros Arjona, V., \& Daponte Codina, A. (2011). Ruido y Salud. (O. (. ANDALUCÍA), Ed.) Andalucía, España: EDITA: Escuela Andaluza de Salud Pública. Consejería de Salud y Bienestar Social. Junta de Andalucía.

Casas Anguita, J., Repullo Labradora, J., \& Donado Campos, J. (19 de Marzo de 2013). La encuesta como técnica de investigación. Elaboración de cuestionarios y tratamiento estadístico de los datos. Investigacioón, 12.

Castro, P. (11 de Marzo de 2005). Universidad de Córdoba. (D. d. Ecologia, Ed.) Recuperado el 16 de Octubre de 2015, de https://www.uco.es/servicios/informatica/windows/filemgr/download/ecolog/Metodos\% 20analisis\%20datos.pdf

De León, M. S. (11 de Septiembre de 2012). Atrapados en el ruido urbano. La Prensa. Obtenido de http://www.prensa.com/politica/Atrapados-ruido-urbano_0_2655484572.html

Decreto 306 (MINSA 04 de Septiembre de 2002). Obtenido de http://docs.panama.justia.com/federales/decretos-ejecutivos/306-de-2002-sep-10-2002.pdf

DECRETO EJECUTIVO 1 (ASAMBLEA LEGISLATIVA 20 de Enero de 2004).

Flores Castro, E., \& Castillo, M. (2012). La Contaminación Acústica (Primera ed.). (P. C. S.A., Ed.) Panamá, Panamá, Panamá: ARTICASA.

Henríquez, F. (2009). Diseño de un Mapa de Ruido en la Ciudad de Panamá utilizando el sofware SoundPlan versión 6.4. Investigación, Universidad Tecnológica de Panamá, Panamá. Obtenido de http://www.fim.utp.ac.pa/documentos/2010/pdf/Resumen_Proyecto_Diseno_Mapa_Rui do_cuidad_de_Panama_1_2009.pdf

Kogan Musso, P. (22 de Julio de 2004). Análisis de la Eficiencia la la Ponderación "A" para evaluar los efectos del Ruido en el Ser Humano. 178. Valdivia, Chile: Universidad Austral de Chile. 
Invest. pens. crit. (ISSN 1812-3864)

Vol. 5, No. 3, septiembre-diciembre 2017

pp. $19-33$

Miyara, F. (28 de Octubre de 2003). NIVELES SONOROS. Recuperado el 3 de Enero de 2017, de http://www.fceia.unr.edu.ar/acustica/biblio/niveles.htm

OMS, (. (1999). GUÍAS PARA EL RUIDO URBANO. (B. Berglund, T. Lindvall, D. Schwela, Edits., Centro Panamericano de Ingeniería Sanitaria, \& Ciencias del Ambiente, Trads.) Ginebra.

Peralta, Á. (14 de Octubre de 2014). Desayuno con fotones. Blog de WordPress.com. Recuperado el 3 de Enero de 2017, de https://bnnas.files.wordpress.com/2014/10/parc3a1metrosonda.png

Publisic Servicios Imagen y Comunicación S.L. (27 de Mayo de 2015). ronahospitalaria.com. Recuperado el 3 de Enero de 2017, de http://www.zonahospitalaria.com/wpcontent/uploads/2015/02/19-1.ThinkstockPhotos-480216217.jpg

Rentería, A. (25 de Abril de 2012). Mucho ruido, oídos sordos. La Prensa. Obtenido de http://impresa.prensa.com/vivir/ruido-oidos-sordos_0_3373162678.html

Ruel, J., Trigueiros-Cunha, N., Puel, J.-L., \& Gil-Loyzaga, P. (27 de Diciembre de 2016). Viaje a Mundo de la Audición. Exploración Funcional. (e. Ligams, Ed.) Francia: Neuorielle. Recuperado el 4 de Enero de 2017, de http://www.cochlea.eu/es/exploracion-funcional

S.F. (11 de Noviembre de 2013). ABC.es. Obtenido de http://www.abc.es/familia-vidasana/20131110/abci-aulas-silencio-201310241724.html

Sánchez, J. E. (04 de Octubre de 2004). Una ciudad enferma por el ruido. Panamá America. Obtenido de http://www.panamaamerica.com.pa/content/una-ciudad-enferma-por-el-ruido

Stevens, G., Flaxman, S., Brunskill, E., Mascarenhas, M., Mathers, C., \& Finucane, M. (24 de Diciembre de 2011). Global and regional hearing impairment prevalence: an analysis of 42 studies in 29 countries. European Journal of Public Health, 23(1).

Universia Panamá. (07 de Agosto de 2012). Universia Panamá. Obtenido de http://noticias.universia.com.pa/en-portada/noticia/2012/08/07/956918/ruidomolesto.html

Villarreal, Y., Castillo, M., Muñoz, A., Toral, J., \& Flores Castro, E. (2013). NIVEL DE RUIDO EN LA CIUDAD DE PANAMÁ. Tecnociencia, 5(2). 